\title{
Traumatic Ophthalmic Artery Pseudoaneurysm Coiled with a Steerable Microcatheter
}

\author{
Michael E. Kelly, David Fiorella
}

Can. J. Neurol. Sci. 2009; 36: 496-499

Traumatic pseudoaneurysms of the ophthalmic artery (OphA) are a rare phenomenon. ${ }^{1,2}$ They can result in subarachnoid hemorrhage if they occur within the dura. Left untreated these lesions are dangerous and have a very high risk of rehemorrhage. In these cases, the acute angulation of the OphA off the internal carotid artery, the proximal location of these intradural lesions and the inability to achieve distal microwire purchase make the catheterization and coil embolization of these lesions challenging.

We utilized a deflectable tip microcatheter for the treatment of this lesion. This publication describes the use of a deflectable tipped microcatheter in a case of a traumatic intradural pseudoaneurysm of the OphA treated by endovascular therapy.

We review the techniques which were applied to take best advantage of the unique properties of this novel microcatheter and facilitate the catheterization and dense coil packing of this difficult aneurysm.

\section{Case Report and Technique}

A 45-year-old previously healthy Caucasian male sustained a base of skull fracture after a fall from a bicycle. He was not wearing a helmet. The patient was a Hunt and Hess Grade IV, and Fisher IV subarachnoid hemorrhage. (Figure 1a) Conventional angiography was performed and a right intradural traumatic OphA pseudoaneurysm was noted. The aneurysm measured $3.4 \mathrm{~mm} \times 2.4 \mathrm{~mm}$ and is shown in Figure $1 \mathrm{~b}$ and $\mathrm{c}$.

During the first unsuccessful attempt a $6 \mathrm{~F}$ Envoy MPD catheter (Cordis Endovascular, Miami Lakes, FL) was used to select the right internal carotid artery and placed at the C2 level. Attempted coiling was then performed by catheterizing the aneurysm with a 0.014 inch Synchro-14 wire, a 0.014 inch Transend EX soft tip wire, a 0.014 inch Transend EX Platinum wire (Boston Scientific, Natick, MA) and a Echelon-10 microcatheter (EV3 Neurovascular, Inc., Irvine, CA) and a straight and 45 degree SL-10 microcatheter (Boston Scientific, Natick, MA) over the various wires. An acute angle at the origin of the OphA prevented the microcatheters from tracking over the wire. A Hyperglide $4 \mathrm{~mm}$ x $20 \mathrm{~mm}$ balloon over an X-pedion 10 wire (EV3 Neurovascular, Inc., Irvine, CA) was navigated just distal to the neck of the aneurysm and the wire placed into the middle cerebral artery. Multiple attempts were made to catheterize the OphA and target aneurysm with the balloon inflated. We were unable to catheterize the aneurysm because of an acute take off of the ophthalmic artery and poor distal wire purchase. We did not perform steam shaping of the microcatheter. The procedure was then stopped.
The patient returned for a second attempted coiling 48 hours later. A 6 F KSAW Shuttle-Select sheath system (Cook, Bloomington, IN) was placed into the right internal carotid artery at the C2 level. This sheath was selected over the 6F Envoy because the inner diameter could accommodate both the Hyperglide balloon and the Enzo catheter. A $4 \mathrm{~mm}$ x $20 \mathrm{~mm}$ Hyperglide balloon and X-pedion 10 wire were navigated just distal to the aneurysm and the wire placed into the middle cerebral artery. A 0.014 inch Synchro-2 wire (Boston Scientific, Natick, MA) was navigated into the aneurysm arising from the proximal aspect of the OphA. The balloon was inflated in an attempt to prevent forward migration of the microcatheter and microwire ("balloon deflection technique") and the deflectable tip catheter was advanced forward. We used the Enzo microcatheter (Micrus Endovascular Corp, San Jose, CA). The Enzo catheter is a 2.8 French dual lumen microcatheter. There is an inner wire that is torqued allowing for deflection of the tip 90 degrees in either direction. At the point of the maximum turn the catheter began to pull the wire from the aneurysm. To counteract this, a second attempt was made with the catheter and it was maximally deflected along the natural curvature of the microwire. In this configuration, the microcatheter tracked over the wire and lodged into the orifice of the OphA. Then, while maintaining gentle forward pressure on the microcatheter, the tip was dynamically deflected both forward and backward with until tactile feedback indicated that it had released and began to move forward over the wire into the OphA (Figures 2a and b, video [on-line]). The aneurysm was completely coiled and deliberate sacrifice of the proximal OphA was performed. During the coil deployment when the microcatheter was pushed back by the coil the tip was deflected and the coil successfully delivered. The microcatheter catheter was straightened and removed from the ophthalmic origin with the balloon inflated at the conclusion of the case. Final angiographic runs were performed and complete coil occlusion of the OphA and pseudoaneurysm was seen. (Figure 3a, b)
From the Division of Neurosurgery, Royal University Hospital (MEK), University of Saskatchewan, Saskatoon, SK, Canada; Departments of Neurosurgery and Neuroradiology, Barrow Neurological Institute (DF), St. Joseph's Hospital and Medical Center, Phoenix, AZ, USA.

Received June 5, 2008. Final Revisions Submitted March 3, 2009. Correspondence to: Michael E. Kelly, Division of Neurosurgery, Royal University Hospital, University of Saskatchewan, Saskatoon, Saskatchewan, S7N 0W8, Canada. 

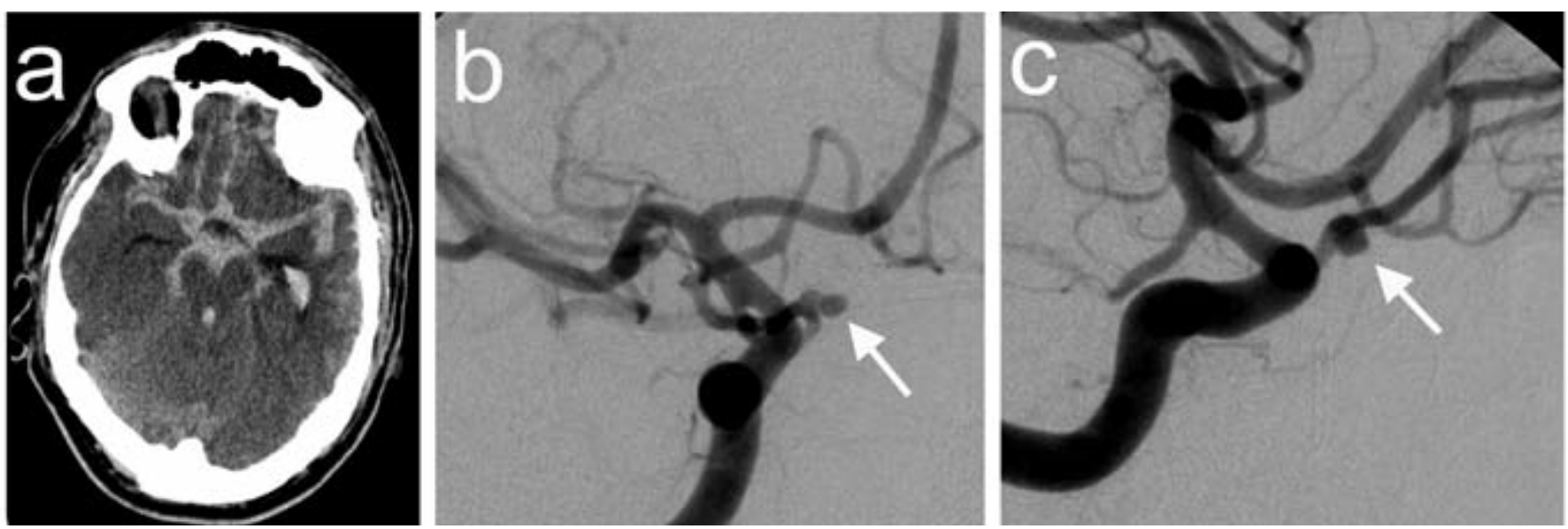

Figure 1: a) Computerized tomography (axial, unenhanced) showing the Fisher IV SAH at the skull base.b) Right anterior oblique (RAO) and (c) lateral projections from a right internal carotid artery angiogram; demonstrate a traumatic, bilobed pseudoaneurysm of the right ophthalmic artery.

\section{Discussion}

Traumatic pseudoaneurysms of the OphA are rare lesions which can cause significant SAH. ${ }^{1,2}$ Sacrifice of the parent vessel is typically the only treatment option. Because of the very fragile nature of the pseudoaneurysm direct catheterization of the aneurysm is treacherous. ${ }^{1}$ The extensive collateral anastamosis from the external carotid artery to the OphA make vessel sacrifice safe with preservation of vision likely. We present our initial experience with adeflectable tip microcatheter for the navigation of the challenging intracranial anatomy of the carotidophthalmic system. (Figure 4)

There are currently no other commercially available microcatheters which allow for dynamic changes in tip configuration to facilitate intracranial navigation. The other available microcatheters are navigated by either wire direction or cerebral blood flow alone.

In the majority of cases, wire direction is sufficient to deliver the microcatheter to the targeted lesion. However, in some cases the over the wire technique is inadequate. This is particularly true when:

1. parent vessel anatomy is tortuous,

2. an abrupt right angle turn must be made off of a larger parent artery into the targeted branch

3. distal microwire access cannot be achieved due to branch vessel tortuosity and/or the presence of an unstable lesion arising from the branch.

All of these factors made catheterization of the OphA challenging in this case. In fact, catheterization could not be
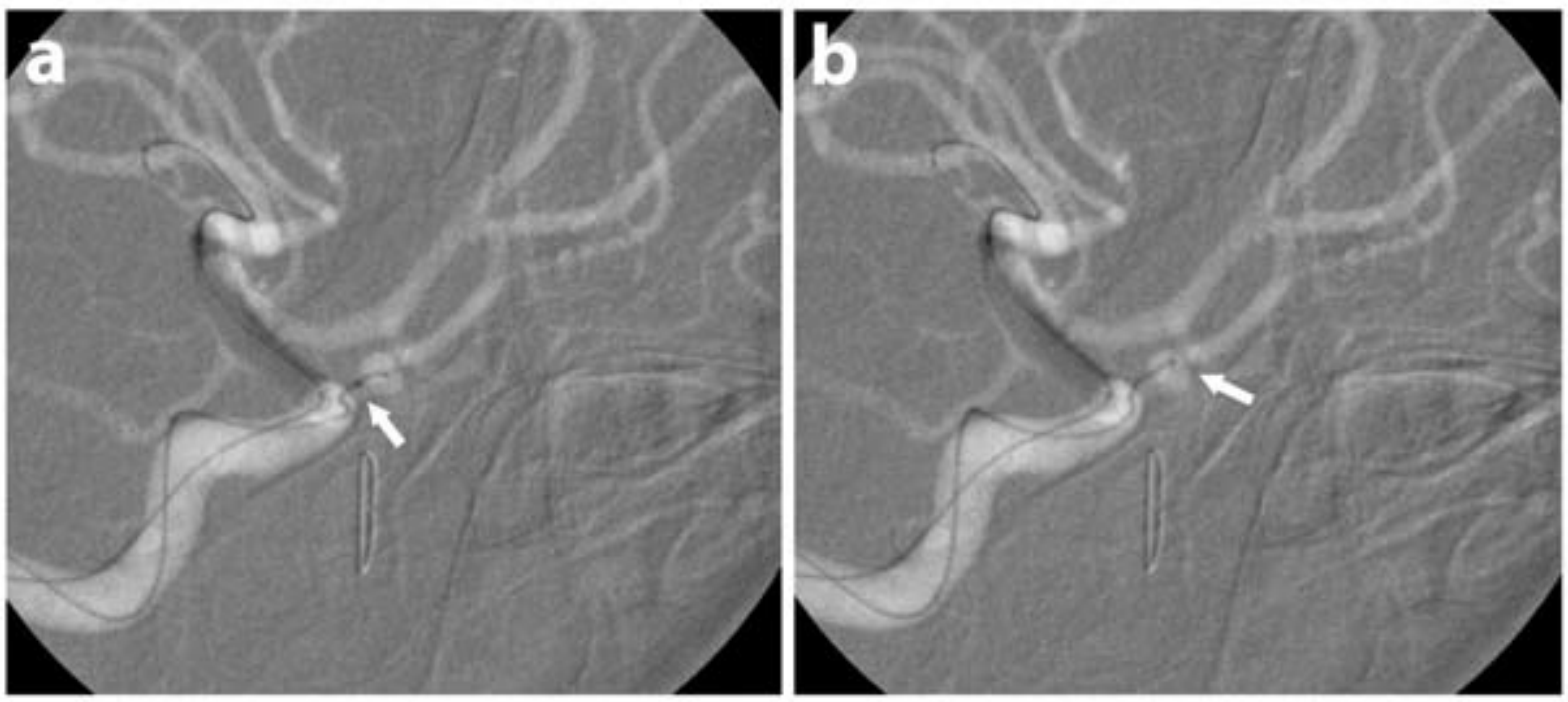

Figure 2: Roadmap images in the RAO (a) and lateral (b) projections depict the Enzo microcatheter within the right internal carotid artery with a 0.014 " Synchro-2 microwire extending from the microcatheter into the ophthalmic artery pseudoaneurysm. A $4 \mathrm{~mm} \times 20 \mathrm{~mm} H \mathrm{Hyperglide}$ balloon is inflated within the supraclinoid ICA to buttress the Enzo microcatheter and prevent distal herniation as it is manipulated into the aneurysm. 

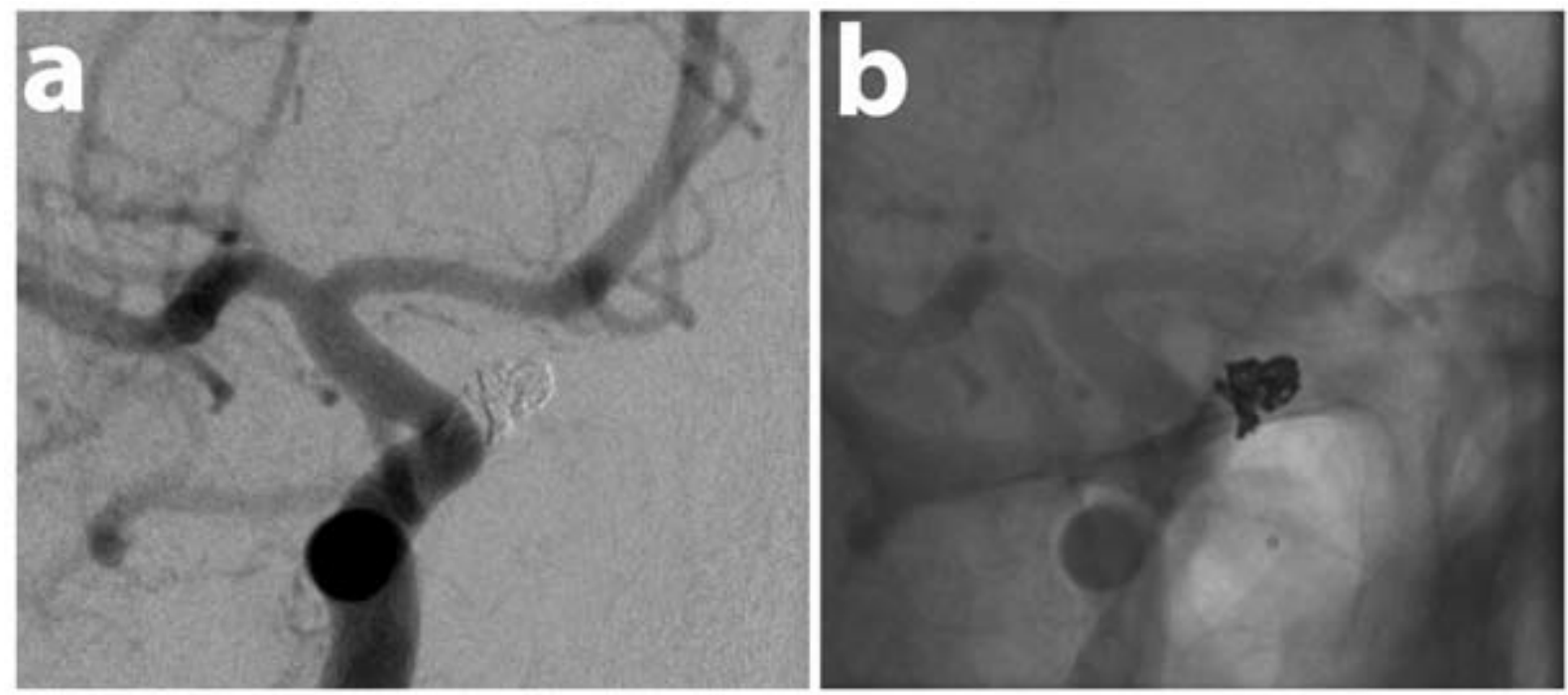

Figure 3: RAO (a) and lateral (b) projections, from a right internal carotid artery angiogram, demonstrate complete deconstructive occlusion of the ophthalmic artery and aneurysm.

achieved despite attempts with multiple different microwires and catheters during our first attempt at embolization and the session was ultimately aborted. However, during the second attempt, the Enzo microcatheter was successfully navigated into the affected segment of the OphA.

Two advantages of the dynamic in vivo shapeability of the microcatheter tip facilitated catheterization. First, during the initial part of the engagement of the OphA by the microcatheter, the microcatheter tip could be deflected to conform to the natural curve of the microwire as it entered the branch artery. This reduced the tension applied to the microwire as the deflectable tip microcatheter was being manipulated over the wire, decreasing the impetus for the microcatheter to drive the microwire out of our tenuous position within the OphA. Second, once engaged within the orifice, while holding gentle steady forward pressure on the proximal microcatheter, the tip shape could be dynamically manipulated through a small radius of curvature back and forth, until tactile feedback and subsequently visualization of catheter movement indicated that the Enzo had started to progress forward into the branch.

Another advantage of this deflectable tip catheter relates to the ability to dynamically deflect the distal tip during coil deployment. In our case, the catheter position was extremely tenuous within the proximal OphA (given the proximal, intradural location of the aneurysm). Because the initial catheterization was so difficult over a microwire, if the catheter was expelled from its position during coil deployment, recatheterization would be extremely challenging and not possible with only the support of a partially delivered embolization coil. Moreover, during the embolization of a pseudoaneurysm,

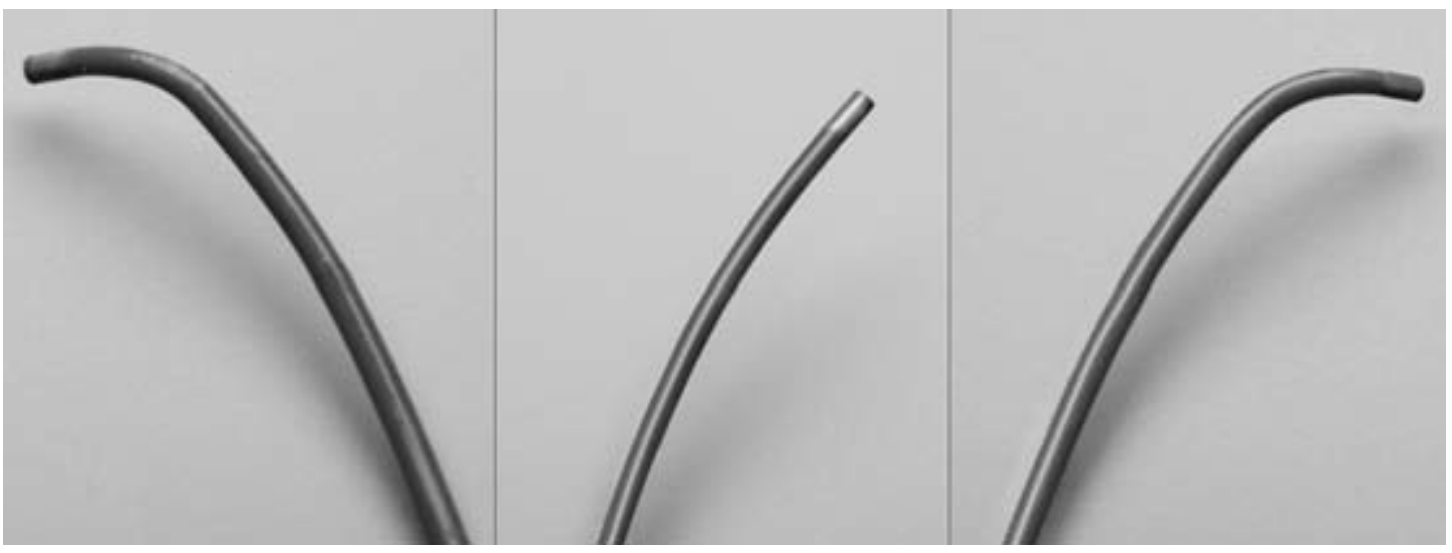

Figure 4: Depiction of the Enzo, deflectable tip microcatheter in maximal deflection (far right and left) and neutral (middle) positions at room temperature. In vivo, at physiological temperatures, the maximal range of deflection is approximately 180 degrees. 
procedural rupture represents a significant hazard. If procedure rupture were encountered, stability within the OphA and the ability to quickly deliver additional embolization coils from this position becomes even more critical.

Thus, during the coil deployment when resistance was felt, the catheter tip could be dynamically deflected with gentle forward pressure maintained on the coil. Eventually, when a release in the resistance to coil delivery was sensed, the tip was maintained in this favorable configuration, and the coil could be delivered without the microcatheter "kicking back" or the operator having to pull the catheter back to allow coil release. This technique of dynamic tip deflection to facilitate coiling was very advantageous in the present case allowing a very dense packing of the lesion and proximal parent artery to achieve therapeutic deconstruction.

There are several appreciable disadvantages of the Enzo microcatheter. The Enzo and a balloon will not fit within a standard $6 \mathrm{~F}$ guide catheter. When using the Enzo with a balloon we prefer to use a $6 \mathrm{~F}$ KSAW Shuttle-Select sheath system (Cook, Bloomington, IN). This catheter is larger and stiffer making access more difficult in some patients. Also, there is a possible increased risk of arterial injury or decreased flow when using this larger system. A second issue is related to the stability of the Enzo within the aneurysm. When the Enzo is fully torqued, it becomes very stable and often will not be push out of the aneurysm. This does seem to improve packing density but there is some increased risk of aneurysm rupture in our opinion.
We present a case of traumatic pseudoaneurysm of the OphA. This was treated with the deflectable tip microcatheter that facilitated the navigation of complex anatomy after more traditional techniques and catheters had failed.

\section{Disclosures}

Drs. Fiorella and Kelly are consultants $(<10 \mathrm{~K})$ for Micrus Endovascular Corp. the maker of the Enzo microcatheter.

\section{REFERENCES}

1. Hopkins JK, Shaibani A, Ali S, Khawar S, Parkinson R, Futterer S, et al. Coil embolization of posttraumatic pseudoaneurysm of the ophthalmic artery causing subarachnoid hemorrhage. Case report. J Neurosurg. 2007;107(5):1043-6.

2. Chun HJ, Yi HJ. Traumatic extracranial pseudoaneurysm on the peripheral ophthalmic artery presenting as delayed intraparenchymal hematoma: case report. Surg Neurol. 2008 Feb 28. 